[Article]

www.chem.pku.edu.cn/whxb

\title{
活化条件对活性碳纳米管比表面积的影响
}

\author{
江 奇* 卢晓英 赵勇 朱晓娅蔡玉冬 钱 兰
}

(西南交通大学材料科学与工程学院, 材料先进技术教育部重点实验室, 超导研究开发中心, 成都 610031)

\begin{abstract}
摘要 以 $\mathrm{KOH}$ 为活化剂, 研究了多壁碳纳米管在制备活性碳纳米管过程中四个重要影响因素: 活化剂用量、活 化温度、活化时间和活化过程中保护气体的流速对所得活性碳纳米管 BET 比表面积的影响并解释了原因. 研究 表明上述四个因素都会对活性碳纳米管的比表面积产生较大的影响, 其中活化剂用量的影响最大, 在研究范围 内可引起比表面积增大约 $241 \mathrm{~m}^{2} \cdot \mathrm{g}^{-1}$. 在这四个影响因素中除活性碳纳米管的比表面积随活化温度的增加而不 断增加外, 其他三个影响因素的变化都会使活性碳纳米管的比表面积出现最大值, 而且四个影响因素的改变, 都 不改变活性碳纳米管的孔洞主要是中孔和大孔的特点.
\end{abstract}

关键词：比表面积, 活性碳纳米管, 活化条件

中图分类号： O647，TM53

\section{Effects of Activation Conditions on the Specific Surface Area of Activated Carbon Nanotubes}

\author{
JIANG, Qi ${ }^{*} \quad$ LU, Xiao-Ying ZHAO, Yong ZHU, Xiao-Tong CAI, Yu-Dong QIAN, Lan \\ (Key Laboratory of Advanced Technologies of Materials of Ministry of Education of China, Superconductivity R\&D Center, \\ School of Materials Science and Engineering, Southwest Jiaotong University, Chengdu 610031, P. R. China)
}

\begin{abstract}
The effects of four important factors, including dosage of activating agent, activation temperature, activation time, and flow rate of protecting gas, on the specific surface area of activated carbon nanotubes (ACNTs) using $\mathrm{KOH}$ as the activating agent were discussed and the possible reasons were proposed. The results showed that the specific surface area of ACNTs could be greatly affected by modifying the four factors amongst which the dosage of activating agent was found to be the dominant one which could result about $241 \mathrm{~m}^{2} \cdot \mathrm{g}^{-1}$ of change in specific surface area. The specific surface area of ACNTs was only continuously enlarged with increasing the activation temperature, but had respective maximum with modifying the other factors in the experiments. The performance of meso-pores and macro-pores of the ACNTs was preserved in the process of modifying the four factors.
\end{abstract}

Keywords: Specific surface area, Activated carbon nanotubes, Activation conditions

碳纳米管 (carbon nanotubes, CNTs) 是在 1991 年发现的一种新型材料 ${ }^{[1]}$, 其独特的纳米中空管结构 使它具有广泛的潜在应用, 如催化剂模板 ${ }^{[2]}$, 量子导 线 ${ }^{[3]}$ 和超级纤维 ${ }^{[4]}$, 所以从其一发现就引起了各界人 士的广泛关注. 到目前为止, 人们已经找到了许多可

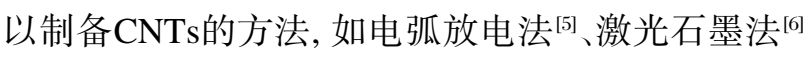
和碳氢化合物催化列解法[]. 但所有的这些方法所制 备的 CNTs 都有一个共同的弱点, 就是 CNTs 的比 表面积不是很大, 特别是多壁碳纳米管, 一般只有 $200 \mathrm{~m}^{2} \cdot \mathrm{g}^{-1[8]}$. 这样就严重影响了CNTs在气体吸附材

Received: June 3, 2005; Revised: July 20, 2005. $\quad{ }^{*}$ Correspondent, E-mail: jiangqi66@ 163.com; Tel: 028-87600720.

国家自然科学基金 (50372052), 四川省科技攻关计划（04GG0363,04GG1661,04GG009-024-07, 05GG009-003) 和西南交通大学科技发展基金 (2004A02)资助项目 
料和电极材料等方面的应用, 因为这些领域都要求 CNTs 具有大的比表面积来容纳气体和电解液, 如 CNTs 用作储氢材料 ${ }^{[9]}$, 其比表面积的大小就具有决 定性的意义.

活性碳纳米管 (activated carbon nanotubes, ACNTs)的提出正是为了弥补CNTs的比表面积不是 很大的弱点 ${ }^{[10]}$, 增强它在需要大比表面积的气体吸 附和电极材料领域的应用. 在前面的研究中 ${ }^{[11]}$, 我们 已经考察了 CNTs 经 $\mathrm{KOH}$ 活化, 得到比表面积更大 的 ACNTs, 并用作电化学超级电容器的电极材料, ACNTs 的电化学容量大大提高, 是原来 CNTs 的 2 倍, 表现出良好的应用前景.

但在后续的研究中, 我们发现经多壁 CNTs 所 制备的 ACNTs 的比表面积的大小与其制备过程中 的活化条件有直接的关系, 活化条件直接影响着 ACNTs 的比表面积的大小. 这一点对于 ACNTs 的 进一步的开发与应用是至关重要的. 为了研究活化 条件如何影响 ACNTs 的比表面积, 我们进一步考 察了 ACNTs 制备过程中活化剂的用量、活化温度、 活化时间和活化过程中保护气体的流速等四个因素 对 ACNTs 比表面积的影响. 下面讨论中若未特别 说明, 碳纳米管均指多壁碳纳米管.

\section{1 实 验}

\section{1 碳纳米管的制备}

实验所用 $\mathrm{CNTs}$, 以 $\mathrm{CH}_{4}$ 为碳源, $\mathrm{La}_{2} \mathrm{NiO}_{4}$ 为催 化剂, 采用碳氢化合物催化裂解法制备 ${ }^{[12]}$. 经硝酸法 纯化, 除去催化剂和杂质后, 烘干待用.

\section{2 活性碳纳米管的制备}

采用管式电阻炉, 以 $\mathrm{KOH}$ 为活化剂进行活化. 将 $\mathrm{KOH}$ 和制备并纯化好的 CNTs 按质量比 $1: 1 、 2$ : $1 、 3: 1 、 4: 1$ 称好, 在玛瑙研钵中充分混合, 然后放置 于陶瓷舟中, 放在水平管式电阻炉中的陶瓷管中. 先 在室温下以流量为 $240 \mathrm{~mL} \cdot \mathrm{min}^{-1}$ 的流速通氮气 $1 \mathrm{~h}$, 再以 $10 \mathrm{~K} \cdot \mathrm{min}^{-1}$ 的升温速度在不同的氮气流速下 $\left(240 、 420 、 860 、 1400 \mathrm{~mL} \cdot \mathrm{min}^{-1}\right)$ 分别升温到 923 、 $1023 、 1123$ 和 $1223 \mathrm{~K}$, 然后分别在各自温度下, 分别 保温 $0.5 、 1 、 2$ 和 $3 \mathrm{~h}$. 最后在氮气的保护下 $(240 \mathrm{~mL}$ $\mathrm{min}^{-1}$ )自然降温到室温, 取出待用.

所得活性碳纳米管分别在二次蒸馏水中搅拌 $24 \mathrm{~h}$ 后, 过滤, 用二次蒸馏水冲洗至呈中性, 在 383 $\mathrm{K}$ 空气中干燥 $48 \mathrm{~h}$, 待用.

\section{3 碳纳米管的表征}

CNTs 和所得 ACNTs 分别采用透射电子显微 镜(TEM)JEOL JEM-100CX, 高分辨透射电子显微镜 (HRTEM, JEOL 2010)进行形貌结构的表征. 采用 AUTOSORP ZXF 4 自动吸附仪 ( $77 \mathrm{~K}, \mathrm{~N}_{2}$ 吸附), 根 据 BET 计算公式进行比表面积和等温吸附曲线的 表征.

\section{2 结果与讨论}

CNTs 和 ACNTs 的 TEM 和 HRTEM 照片与前 面的报道很相似, 请参见文献[11]. 总之, 碳纳米管经 活化处理后, 两端封口打开, 长度变短, 管壁变得不 光滑(管壁上有孔出现), 但其独特的纳米中空管结构 却得到了保存. 当然, 还有一些碳纳米管的碎片存 在, 这些碳纳米管碎片和上面的长度变短、管壁变得 不光滑的碳纳米管共同组成活性碳纳米管.

\section{1 活化剂用量的影响}

为研究活化剂的用量对 ACNTs 的比表面积的 影响, 我们将其他的影响因素固定不变, 而只改变 $m(\mathrm{KOH}): m(\mathrm{CNTs}$ )的比例(分别为 $1: 1 、 2: 1 、 3: 1 、 4: 1$ ). 因 此, 在这节的活化实验中, $\mathrm{N}_{2}$ 的流量、活化温度和活 化时间分别固定为 $420 \mathrm{~mL} \cdot \mathrm{min}^{-1} 、 1023 \mathrm{~K}$ 和 $1 \mathrm{~h}$.

图 $1 \mathrm{a}$ 是ACNTs的氮气等温吸附曲线随 $m(\mathrm{KOH})$ : $m(\mathrm{CNTs})$ 比例的增加的变化关系. 由此图可知, 随着 $m(\mathrm{KOH}): m(\mathrm{CNTs})$ 比例的增加, ACNTs 的吸附等温 线在图中的位置逐渐上升, 表明氮气吸附容量逐 渐增加. 当 $m(\mathrm{KOH})$ : $m(\mathrm{CNTs})$ 的比例增加为 3 时, ACNTs 的氮气吸附容量达到一个最大值. 而且从这 些等温吸附曲线的走势来看, 他们基本属于 II 和 IV 型吸附曲线 ${ }^{[13]}$, 说明得到的 ACNTs 所含的孔主要是 中孔和大孔. ACNTs 吸附量的增加, 意味着 ACNTs 的比表面积的增加.

图 $1 b$ 就是ACNTs 的BET 比表面积随 $m(\mathrm{KOH})$ : $m(\mathrm{CNTs})$ 比例的变化的关系图. 由图可知, 所得 ACNTs 的比表面积都大于未经活化的 CNTs 的比 表面积 $\left(180.9 \mathrm{~m}^{2} \cdot \mathrm{g}^{-1}\right)$; 而且随着 $m(\mathrm{KOH}): m(\mathrm{CNTs})$ 比例的增加, ACNTs 的比表面积迅速增加, 当 $m(\mathrm{KOH}): m(\mathrm{CNTs})$ 比例增加为 3 时, 其比表面积达 到最大值 $\left(511.7 \mathrm{~m}^{2} \cdot \mathrm{g}^{-1}\right)$. 若 $m(\mathrm{KOH}): m(\mathrm{CNTs})$ 的比例 进一步增加, ACNTs 的比表面积不但不会增加, 反 而下降.

我们认为, CNTs 管壁上有成千上万的碳原子, 理论上讲, 碳是以 $s p^{2}$ 杂化与其他碳一起, 组成六元 环结构进而形成管状结构. 但实际上由于制备过程 

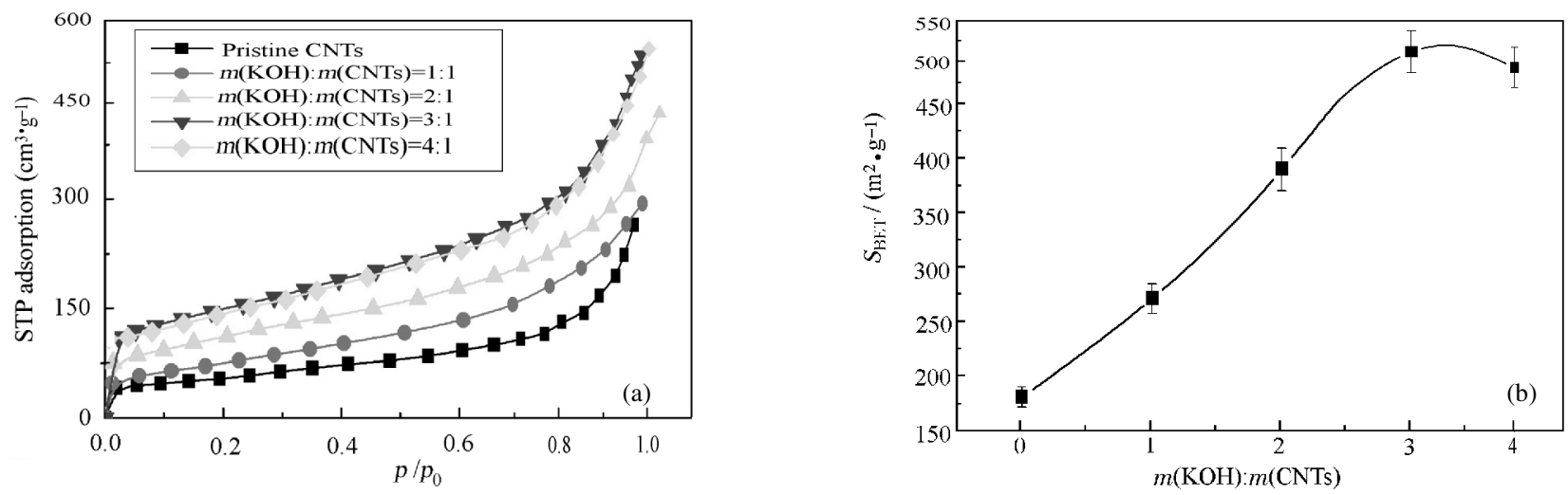

图 1 活化剂用量对活性碳纳米管等温吸附曲线(a)和 BET 比表面积(b)的影响.

Fig.1 Effects of mass ratio of KOH to CNTs on ACNTs adsorption isotherms (a) and BET specific surface area (b) the $\mathrm{N}_{2}$ flow rate, activation temperature, and activation time keeping as $420 \mathrm{~mL} \cdot \mathrm{min}^{-1}, 1023 \mathrm{~K}$, and $\mathrm{l} \mathrm{h}$

中的种种影响因素, CNTs 上的碳不全是六元环, 还 有五元环和七元环等, 如 CNTs 的弯曲点和两端的 封口处的碳等. 而由于张力作用, 六元环的稳定性优 于五元环和七元环, 所以 $\mathrm{KOH}$ 和 CNTs 的反应, 首 先在五元环和七元环上的碳上进行, 因此 CNTs 的 弯曲点和两端的封口处的碳首先发生反应, 随着活 化的进行, 进而将 CNTs 的两端的封口打开, 并在 CNTs 的弯曲处折断. CNTs 两端封口的打开可以让 气体进人 CNTs 的内腔和层间空隙, 有利于比表面 积的提高; CNTs 在弯曲处折断有利于 CNTs 相互堆 积所造成的孔的生成 ${ }^{[14]}$, 同样有利于比表面积的提 高. 当然那些由于活化过度产生的 CNTs 碎片, 同样 由于相互堆积, 有利于比表面积的提高. 若活化程度 不够, 就只能在 CNTs 的管壁上留下孔洞 ${ }^{[10]}$, 同样有 利于比表面积的提高. 随着 $\mathrm{KOH}$ 用量的增加, CNTs 管壁上的孔的孔径将随着反应的进行而不断增大, 即达到一定程度后, 管径较小的孔之间就相互连接,
组成管径较大的孔, 从而造成比表面积的下降 ${ }^{[10]}$. 当 然由于 ACNTs 相互堆积所生成的孔对比表面积的 贡献, 也有一最佳值 ${ }^{[14]}$. 所以在图 $1 \mathrm{~b}$ 上, ACNTs的比表 面积随着 $\mathrm{KOH}$ 用量的增加会有一最大值.

\section{2 保护气体流速的影响}

在本研究中, 所使用的保护气体都是氮气 (99.99\%). 就一般情况而言, 氮气是作为保护气体, 其主要作用是使 CNTs 在活化过程中, 免受空气中 的氧气氧化. 但在实验中, 我们发现氮气的流速同样 也会影响 ACNTs 的比表面积的变化. 为严格考察 氮气对 ACNTs 比表面积的影响, 我们只改变氮气 流速的大小, 分别为 $240 、 420 、 860$ 和 $1400 \mathrm{~mL} \cdot \mathrm{min}^{-1}$, 而其他条件如 $m(\mathrm{KOH}): m(\mathrm{CNTs})$ 比例、活化温度和 活化时间分别固定为 $2: 1 、 1023 \mathrm{~K}$ 和 $1 \mathrm{~h}$.

图 $2 \mathrm{a}$ 是所得 ACNTs 的等温吸附曲线随保护 气体 $\mathrm{N}_{2}$ 流速变化的曲线图. 由此图可知, ACNTs 的 等温吸附曲线随 $\mathrm{N}_{2}$ 流速的变化, 而发生变化; 特别

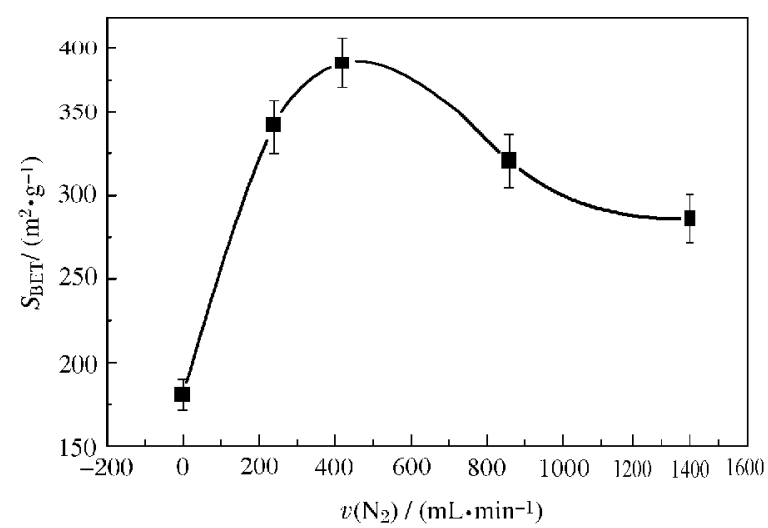

图 2 保护气体流速对活性碳纳米管等温吸附曲线(a)和 BET 比表面积(b)的影响.

Fig.2 Effects of $\mathrm{N}_{2}$ flow rate on ACNTs adsorption isotherms (a) and BET specific surface area (b) the ratio of $m(\mathrm{KOH}): m(\mathrm{CNTs})$, activation temperature, and activation time keeping as $2: 1,1023 \mathrm{~K}$, and $1 \mathrm{~h}$ 
是相对压力 $\left(p / p_{0}\right)$ 大于 0.85 以后, 曲线的走势与 IV 型 等温吸附曲线更加相似 ${ }^{[13]}$. 图 $2 b$ 是所得ACNTs的比 表面积随保护气体 $\mathrm{N}_{2}$ 变化的曲线图. 图中曲线表 示, 刚开始时, ACNTs 的 BET 比表面积随着 $\mathrm{N}_{2}$ 流 速的增大而增大, 当 $\mathrm{N}_{2}$ 流速增大到 $420 \mathrm{~mL} \cdot \mathrm{min}^{-1}$ 时, 比表面积达到最大值 $\left(389.2 \mathrm{~m}^{2} \cdot \mathrm{g}^{-1}\right)$; 然后, 随着 $\mathrm{N}_{2}$ 流 速的进一步增大, ACNTs的比表面积开始下降.

结合活性碳的活化过程 ${ }^{[15]}$, 我们认为 ACNTs 的 制备过程与活性碳的活化过程相似, 都是在高温下 以气态的形式存在, 所以保护气体 $\left(\mathrm{N}_{2}\right)$ 流速大小将 影响活化的微环境. 在我们的实验范围内, 随着 $\mathrm{N}_{2}$ 流速的增加, 活化效果有一最佳值出现, 这正如图 $2 b$ 中所示的 ACNTs 的比表面积随 $\mathrm{N}_{2}$ 流速的增加 有一最大值.

\section{3 活化温度的影响}

在活化过程中, 就一般而言, 活化温度对于活化 效果的影响具有举足轻重的地位. 为研究活化温度 对 ACNTs 比表面积的影响, 固定 $m(\mathrm{KOH}): m(\mathrm{CNTs})$ 的比例、保护气体 $\mathrm{N}_{2}$ 流速和活化时间分别为 $2: 1$ 、 $420 \mathrm{~mL} \cdot \mathrm{min}^{-1}$ 和 $1 \mathrm{~h}$, 而活化温度从室温到 $1223 \mathrm{~K}$ 进行变动.

图 3 是 ACNTs 的 BET 比表面积随活化温度的 变化曲线. 由图可知, ACNTs 的 BET 比表面积随着 活化温度的增加而不断增加, 表明活化温度越高, 活 化效果越好, 活化程度就越深人. 这点和 $\mathrm{KOH}$ 作为 活化剂对碳系列进行活化的情况是一致的 ${ }^{[15]}$, 更高的 活化温度对应着更大的比表面积. 当活化温度从 $923 \mathrm{~K}$ 升到 $1223 \mathrm{~K}$ 时, 所得 ACNTs 的 BET 比表面

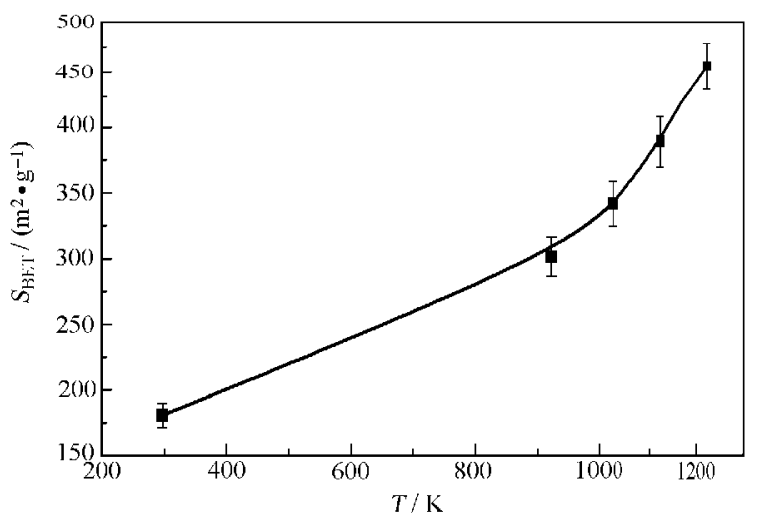

图 3 活化温度对活性碳纳米管 BET 比表面积的影响

Fig.3 Effects of activation temperature on ACNTs BET specific surface area

the ratio of $m(\mathrm{KOH}): m(\mathrm{CNTs}), \mathrm{N}_{2}$ flow rate, and activation time keeping as $2: 1,420 \mathrm{~mL} \cdot \mathrm{min}^{-1}$, and $1 \mathrm{~h}$
积从 $301.5 \mathrm{~m}^{2} \cdot \mathrm{g}^{-1}$ 增大到 $455.6 \mathrm{~m}^{2} \cdot \mathrm{g}^{-1}$ (至于这些 ACNTs 的等温吸附曲线, 由于和前面的走势一致, 这里未给出).

\section{4 活化时间的影响}

在这个研究中, 活化时间从 0 到 $3.0 \mathrm{~h}$ 之间变 化, 而其他活化条件, 如 $m(\mathrm{KOH}): m(\mathrm{CNTs})$ 的比例、 保护气体 $\mathrm{N}_{2}$ 的流速及活化温度分别控制为 $2: 1 、 420$ $\mathrm{mL} \cdot \mathrm{min}^{-1}$ 和 $1023 \mathrm{~K}$.

图 4 为 ACNTs 的 BET 比表面积随活化时间变 化的关系曲线图. 由图可知, ACNTs 的 BET 比表面 积刚开始时随着活化时间的增加, 比表面积增加, 但 当活化时间增加到 $2 \mathrm{~h}$ 时, 其比表面积达到一最大 值, 然后其比表面积随活化时间的进一步增加, 出现 一小幅的下降 (也可看成一平台, 至于这些 ACNTs 的等温吸附曲线, 由于和前面的走势一致, 这里未给 出).

我们认为, 随着活化时间的进行, 活化剂 $\mathrm{KOH}$ 及其分解的有效活化成分 (如 $\mathrm{K}_{2} \mathrm{O}$ 和 $\mathrm{H}_{2} \mathrm{O}$ ) 渐渐被反 应消耗, 到 $2.0 \mathrm{~h}$ 时, 基本用完, 这时 ACNTs 的比表 面积达到最大值. 然后, 随着活化时间的进一步延 长, ACNTs 上生成的孔之间在高温下进行缓慢地重 整, 导致一部分孔合并成大的孔, 从而出现比表面积 的微小下降.

\section{5 四个影响因素的比较}

由上面的研究知道, 在 ACNTs 的制备过程中, 四个重要的影响因素: 活化剂的用量、活化温度、活 化时间和活化过程中保护气体的流速等都会不同程 度地影响所得 ACNTs 的 BET 比表面积的大小. 为

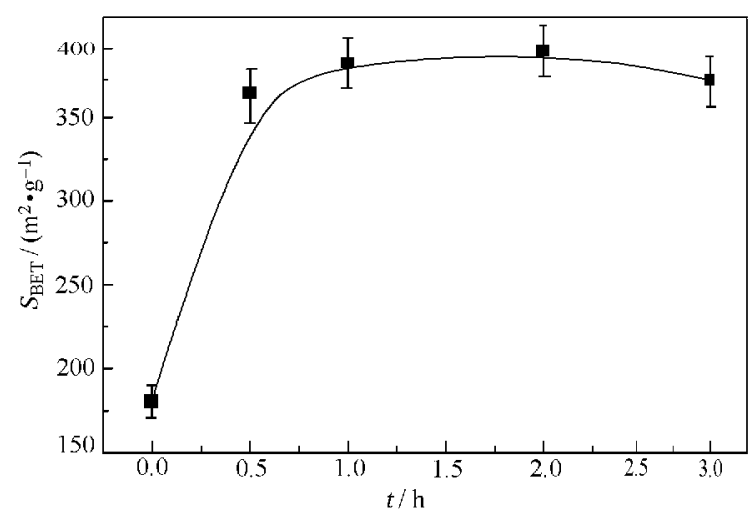

图 4 活化时间对活性碳纳米管 BET 比表面积的影响

Fig.4 Effects of activation time on ACNTs BET specific surface area

the ratio of $m(\mathrm{KOH}): m(\mathrm{CNTs})$, activation temperature, and $\mathrm{N}_{2}$ flow rate keeping as $2: 1,1023 \mathrm{~K}$, and $420 \mathrm{~mL} \cdot \mathrm{min}^{-1}$ 


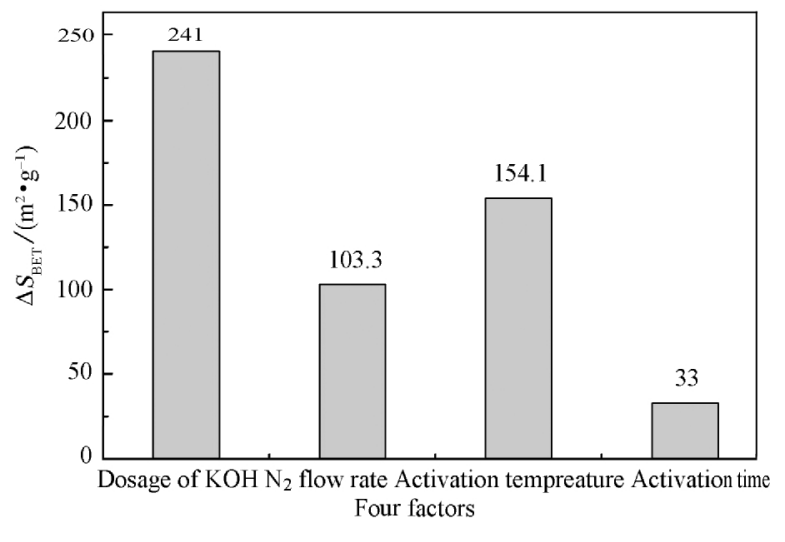

图 5 四个影响因素分别对活性碳纳米管 BET 比表面积 的影响

Fig.5 Effects of four factors on the ACNTs BET specific surface area

研究这四个影响因素中, 那些因素的影响较大, 那些 较小, 我们将它们对 ACNTs 比表面积的影响列在 图 5 中进行比较.

由图 5 可知, 在四个影响因素中, 活化剂的用量 $(m(\mathrm{KOH}) / m(\mathrm{CNTs})$ 的比例)对 ACNTs 的比表面积的 影响最大, 达到约 $241 \mathrm{~m}^{2} \cdot \mathrm{g}^{-1}$, 其次是活化温度, 达到 $154.1 \mathrm{~m}^{2} \cdot \mathrm{g}^{-1}$; 影响最小的是活化时间, 只有约 33 $\mathrm{m}^{2} \cdot \mathrm{g}^{-1}$.

\section{3 结 论}

详细研究了在活性碳纳米管制备过程中, 四个 重要的影响因素: 活化剂的用量、活化温度、活化时 间和活化过程中保护气体的流速等对所得 ACNTs 的比表面积的影响, 并解释了原因. 发现, 上述四个 影响因素都对 ACNTs 的比表面积有较大的影响作 用, 其中活化剂用量的影响最大, 可导致约 $241 \mathrm{~m}^{2}$ • $\mathrm{g}^{-1}$ 的变化. 在我们的研究范围内, 除 ACNTs 的比表
面积随活化温度的增加而不断增加外, 其他三个影 响因素都出现最大值. 而且这些影响因素的调整, 都 不会改变 ACNTs 的孔洞主要是中孔和大孔的特性, 为进一步的应用研究打下基础.

\section{References}

1 Iijima, S. Nature, 1991, 354: 56

2 Penza, M.; Antolini, F.; Vittori-Antisari, M. Thin Solid Films, 2005, 472(1-2): 246

3 Teredesai, P. V.; Sood, A. K.; Muthu, D. V. S; Sen, R.; Govindaraj, A.; Rao, C. N. R. Chem. Phys. Lett., 2000, 319(3-4): 296

4 Ajayan, P. M.; Iijima, S. Nature, 1993, 361(6410): 333

5 Ebbesen, T. W.; Ajayan, P. M. Nature, 1992, 358(6383): 220

6 Morales, A. M.; Lieber, C. M. Science, 1998, 279: 208

7 Mukhopadhyay, K.; Koshio, A.; Sugai, T.; Tanaka, N.; Shinohara, H.; Konya, Z. Nagy, J. B. Chem. Phys. Lett., 1999, 303(1-2): 117

8 Baughman, R. H.; Zakhidov, A. A.; Heer, W. A. Science, 2002, 297: 787

9 Zhu, H. W.; Xu, C. L.; Chen, A.; Mao, Z. Q.; Wei, B. Q.; Liang, J.; Wu, D. H. Carbon Tech., 2000, 109(4): 12 [朱宏伟, 徐才录, 陈 桉, 毛宗强, 魏秉庆, 梁 吉, 吴德海. 炭素技术(Tansu Jishu), 2000, 109(4): 12]

10 Jiang, Q.; Qu, M. Z.; Zhang, B. L.; Yu, Z. L. Carbon, 2002, 40 (14): 2743

11 Jiang, Q.; Lu, X. Y.; Zhao, Y.; Yu, Z. L. Acta Phys. -Chim. Sin., 2004, 20(5): 546 [江 奇, 卢晓英, 赵 勇, 于作龙. 物理化学 学报(Wuli Huaxue Xиebao), 2004, 20(5): 546]

12 Liang, Q.; Gao, L. Z.; Li, Q.; Tang, S. H.; Liu, B. C.; Yu, Z. L. Carbon, 2001, 39(6): 897

13 Sing, K. S. W.; Everett, D. H.; Haul, R. A. W.; Moscou, L.; Pierotti, R. A.; Rouquerol, J.; Siemieniewska, T. Pure \& Appl. Chem., 1985, 57(4): 603

14 Yang, Q. H.; Hou, P. X.; Bai, S.; Wang, M. Z.; Cheng, H. M. Chem. Phys. Lett., 2001, 345: 18

15 Lillo-Rodenas, M. A.; Cazorla-Ameros, D.; Linares-Solano, A., Carbon, 2003, 41(2): 267 